Kompass

Onkologie

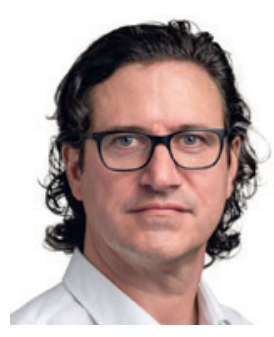

\section{Martin Pölcher}

Rotkreuzklinikum München Frauenklinik, München, Deutschland

\title{
Zervix- und Endometriumkarzinom: Den Überblick behalten
}

Der Einsatz von Checkpoint-Inhibitoren bei gynäkologischen Malignomen wird in klinischen Studien noch intensiv untersucht. Aber es ist absehbar, dass sich CheckpointInhibitoren in bestimmten Therapiesituationen in unterschiedlicher Form als Standard etablieren werden - als Ergänzung zur Polychemotherapie, als alternative Monotherapie oder mit weiteren zielgerichteten Substanzen gemeinsam.

Es gilt den Überblick zu behalten. Nicht nur laufen erfreulicherweise viele Studien, sondern es erscheinen auch Mehrfachpublikationen aus «ongoing Phase Ib/II Studies». Und neuerdings erfolgen Zulassungen auch aufgrund der Ergebnisse von BasketTrials (FDA-Zulassung von Pembrolizumab bei MSI-Tumoren) oder Phase-I-Studien (Dostarlimab, s.u.).

Bei Patientinnen mit Endometriumkarzinom oder Zervixkarzinom stehen folgende Therapien im Mittelpunkt:

- Pembrolizumab als zusätzliches Medikament zur Kombination der Platin-Taxanhaltigen Chemotherapie mit der antiangiogenetischen Erhaltungstherapie Bevacizumab (Studie Keynote-826);

- Pembrolizumab in Kombination mit dem Multityrosinkinasehemmer Lenvantinib bei Patientinnen mit rezidiviertem oder metastasiertem Endometriumkarzinom nach platinhaltiger Vortherapie-als AlternativezurChemotherapie (Keynote 146 und aktuell auch neue Daten der Phase-III-Studie Keynote-775);
- Sowie der bereits zugelassene PD-L1Antikörper Dostarlimab, ebenfalls bei Patientinnen mit rezidiviertem oder metastasiertem Endometriumkarzinom nach platinhaltiger Vortherapie und Nachweis eines Tumors mit Mikrosatelliteninstabilität.

Die Immuntherapie etabliert sich also in der Gynäkologischen Onkologie zuerst in Therapiesituationen, in denen Chemotherapeutika aufgrund niedriger Ansprechraten und kurzer Wirkdauer enttäuschten. Allerdings trifft dies nicht bei allen Entitäten gleichermaßen zu: Beim high-grade Ovarialkarzinom haben die Checkpoint-Inhibitoren bei Platinresistenz bislang nicht überzeugt und Studien prüfen den Einsatz in Kombination mit mehreren weiteren Therapien wie PARP-Inhibitoren, Antiangiogenese etc.

Nach wie vor ist beim Endometriumkarzinom eine platinbasierte Erstlinientherapie vorgesehen, Carboplatin und Paclitaxel haben die Anthrazykline verdrängt. Spannend ist die chemotherapiefreie adjuvante Checkpoint-Inhibitor Therapie, die in Phase-III-Studien nun untersucht wird.

Die Indikationsstellung für eine adjuvante Therapie (Strahlentherapie/Chemotherapie/Kombination aus beidem) ist beim Endometriumkarzinom aus den Einschlusskriterien mehrerer klinischer Studien abgeleitet, über die verschiedene Risikogruppen definiert werden. Diese komplizierte Risikoeinteilung in low risk, intermediate, high- 
intermediate und high risk wurde aktuell angepasst und in den Consensus Guidelines der Fachgesellschaften ESGO, ESTRO und ESP publiziert. Erstmals wird hierbei die molekulare Subklassifikation der Endometriumkarzinome berücksichtigt, die sich aus den Untersuchungen des Cancer Genome Atlas ergeben haben. Sie löst die bisherige Einteilung in Typ-I und Typ-II Karzinome durch die neuen klinischen Subtypen ab. Diese werden kategorisiert in POLE-mutierte, mikrosatelliteninstabile oder p53-positive Tumoren, sowie Tumoren mit nicht-spezifizierbarem molekularen Profil (NSMP).

Noch bestehen keine klaren Empfehlungen, welche Tumoren komplett untersucht werden sollen. Auch aus Kostengründen ist eine Subklassifizierung aller Tumoren derzeit nicht realisierbar. Die Immunhistochemie und die POLE-Mutationsanalyse sind bei allen high-grade und allen high risk Tumoren bei der Erstdiagnose wünschenswert, da hier adjuvante Therapien vorgesehen sind, die durch die Subklassifizierung präziser bestimmt werden können. Im Rezidivfall oder bei Metastasierung ist aufgrund der neuen Immuntherapien eine Testung obligat.

Die neu vorgestellte Einteilung der Risikogruppen beim Endometriumkarzinom ist im Detail sinnvoll, birgt aber auch Gefahren: Die p53-positiven Tumoren mit Muskelinvasion, die als pT1a G1/G2 klassifiziert sind, werden jetzt zur high risk Gruppe gezählt, die eine adjuvante Chemotherapie erhalten sollen. Für eine solch weitreichende Empfehlung fehlen aber noch klinische Daten.

Für das onkologische Gesamtkonzept ist die operative Therapie von überragender Bedeutung und wichtige Fragestellungen werden aktuell diskutiert.

Die radikale Chirurgie, wie z.B. die Wertheim Operation beim Zervixkarzinom oder die systematische Lymphonodektomie beim Endometriumkarzinom, ist aufgrund ihrer Langzeitmorbidität auf dem Prüfstand. Müssen die Parametrien beim Zervixkarzinom unter $2 \mathrm{~cm}$ entfernt werden? Wie zuverlässig ist die Sentinel-Lymphonodektomie bei Zervix- und Endometriumkarzinom? Ist ein Uterusmanipulator schädlich?
Die überraschenden Daten der randomisierten LACC Studie zur radikalen Hysterektomie dürften noch nachhallen, so meint man zumindest. Das Outcome von minimal-invasiv operierten Patientinnen war desaströs im Vergleich zu denen, die eine Laparotomie erhielten. Vergleichbare Ergebnisse haben sich auch in vielen Ländern in retrospektiven Daten der Krebsregister oder in Umfragen in ähnlicher Dimension gezeigt. Umso erstaunlicher, dass offenbar über 40\% der befragten Kliniken in Deutschland am minimal-invasiven Verfahren festhalten.

In naher Zukunft kann man hoffen, dass bei der Therapie des Endometriumkarzinoms die Subgruppen zu weiteren spezifischen Therapieeinsätzen führen können, so z.B. die endokrine Therapie (oder wie in der PALEO Studie in Kombination mit Palbociclib). Beim Zervixkarzinom sind weitere immunologische Therapieansätze in klinischer Prüfung wie z.B. eine therapeutische Vakzinierung gegen HPV-Viren in Kombination mit Checkpoint-Inhibitoren. 